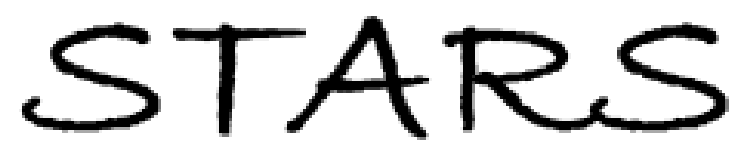

University of Central Florida

STARS

$1-1-2013$

\title{
Atomic-scale simulation of the thermodiffusion of hydrogen in palladium
}

\author{
William C. Tucker \\ University of Central Florida \\ Lalit Shokeen \\ University of Central Florida \\ Patrick K. Schelling \\ University of Central Florida
}

Find similar works at: https://stars.library.ucf.edu/facultybib2010 University of Central Florida Libraries http://library.ucf.edu

This Article is brought to you for free and open access by the Faculty Bibliography at STARS. It has been accepted for inclusion in Faculty Bibliography 2010 s by an authorized administrator of STARS. For more information, please contactSTARS@ucf.edu.

\section{Recommended Citation}

Tucker, William C.; Shokeen, Lalit; and Schelling, Patrick K., "Atomic-scale simulation of the thermodiffusion of hydrogen in palladium" (2013). Faculty Bibliography 2010s. 4779.

https://stars.library.ucf.edu/facultybib2010/4779

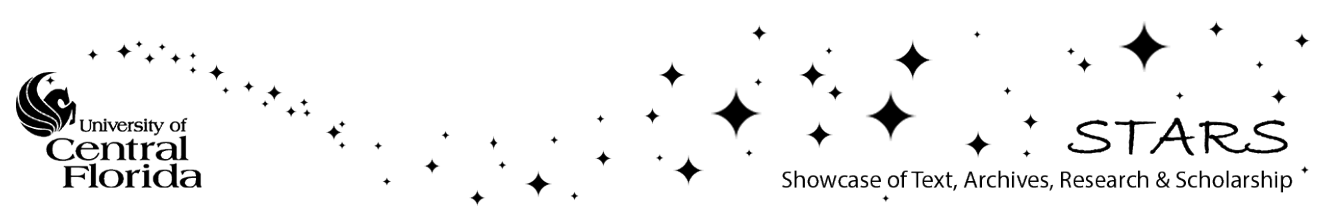




\section{Atomic-scale simulation of the thermodiffusion of hydrogen in palladium}

Cite as: J. Appl. Phys. 114, 063509 (2013); https://doi.org/10.1063/1.4816961

Submitted: 14 February 2013 . Accepted: 15 July 2013 . Published Online: 09 August 2013

William C. Tucker, Lalit Shokeen, and Patrick K. Schelling
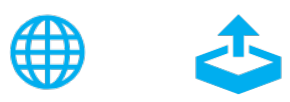

\section{ARTICLES YOU MAY BE INTERESTED IN}

Molecular-dynamics calculation of the vacancy heat of transport

Journal of Applied Physics 116, 023506 (2014); https://doi.org/10.1063/1.4886577

Thermodynamics and kinetics of silicon under conditions of strong electronic excitation Journal of Applied Physics 109, 073503 (2011); https://doi.org/10.1063/1.3554410

Analysis of simulation methodology for calculation of the heat of transport for vacancy thermodiffusion

Journal of Applied Physics 116, 023504 (2014); https://doi.org/10.1063/1.4887121

\section{Applied Physics Reviews} Now accepting original research

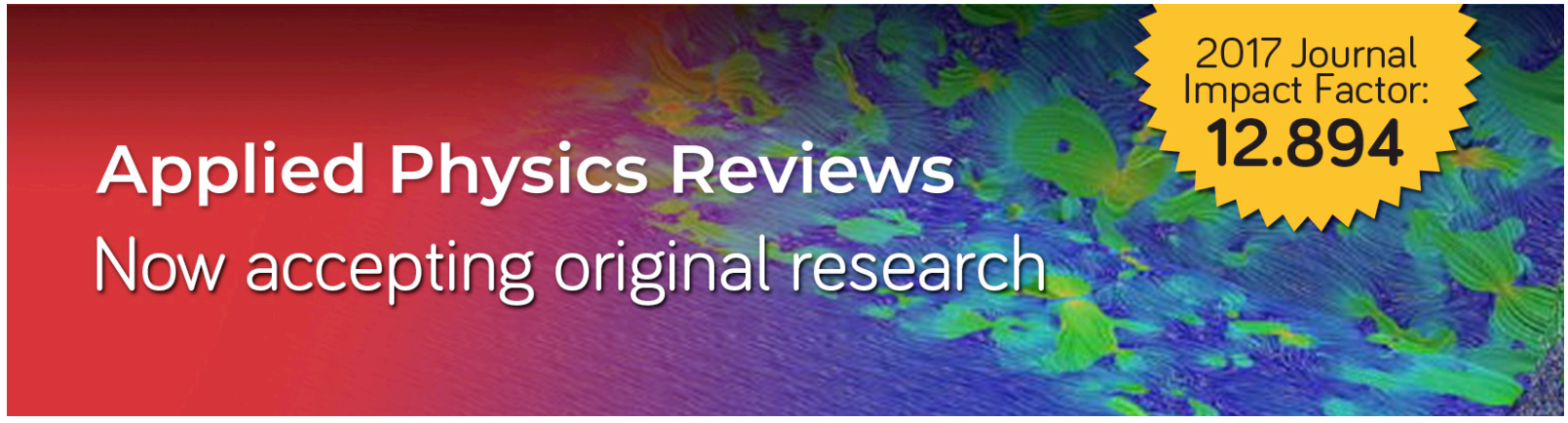




\title{
Atomic-scale simulation of the thermodiffusion of hydrogen in palladium
}

\author{
William C. Tucker, ${ }^{1}$ Lalit Shokeen, ${ }^{2}$ and Patrick K. Schelling ${ }^{1,2}$ \\ ${ }^{1}$ Department of Physics, University of Central Florida, 4000 Central Florida Blvd., Orlando, \\ Florida 32816, USA \\ ${ }^{2}$ Advanced Material Processing and Analysis Center, University of Central Florida, \\ 4000 Central Florida Blvd., Orlando, Florida 32816, USA
}

(Received 14 February 2013; accepted 15 July 2013; published online 9 August 2013)

\begin{abstract}
We report molecular-dynamics simulations of $\mathrm{Pd}: \mathrm{H}$ to elucidate transport properties, with special focus placed on determining the temperature dependence of the heat of transport $Q^{*}$. Simulation results are analyzed using the Green-Kubo approach. It is found that $Q^{*}$ describing the thermodiffusion of hydrogen increases linearly with temperature. By contrast, the reduced heat of transport $Q^{* \prime}=Q^{*}-h_{2}$, with $h_{2}$ the partial enthalpy of hydrogen, is approximately temperature independent. By computing separately the potential, kinetic, and virial contributions to $Q^{*}$, it is possible to understand key features of the thermodiffusion process. In particular, the sum of the kinetic and potential energy of hydrogen atoms is increased above that of an average hydrogen atom by an amount comparable to the migration energy during a successful hop. However, the virial term in the energy flux is less than what would be expected based on the average local stress contribution due to the hydrogen atoms. Detailed calculations show that the relevant component of the stress tensor due to a hopping hydrogen atom exhibits a minimum at the transition state. Hence, while $Q^{*}$ has significant positive contributions due to the excited nature of the hopping hydrogen atom, the reduced heat of transport $Q^{* \prime}$ can still be negative. The results here present important insight into the failure of simple kinetic theories of thermodiffusion, and provide a new perspective that can be tested on other systems. (C) 2013 AIP Publishing LLC. [http://dx.doi.org/10.1063/1.4816961]
\end{abstract}

\section{INTRODUCTION}

We recently reported on the application of the GreenKubo method to analyze molecular-dynamics simulations of the $\mathrm{Pd}: \mathrm{H}$ system. ${ }^{1}$ The principle objective was to compute the reduced heat of transport $Q^{* \prime}$ which determines both the magnitude and direction of the hydrogen flux in the presence of a temperature gradient. This problem has previously also been studied by Gillan using molecular-dynamics (MD) simulation. $^{2,3}$ It was demonstrated in Ref. 1 that the simulation model predicts diffusion of hydrogen from low to high temperatures with $Q^{* \prime} \approx-0.21 \mathrm{eV}$ at the simulation temperature $T=980 \mathrm{~K}$. However, the results themselves shed very little light on the thermodiffusion phenomenon, which has only been described at the level of phenomenological transport theory.

In this article, we revisit the problem of thermodiffusion of hydrogen in a palladium lattice. In addition to results at $T=980 \mathrm{~K}$ previously reported in Ref. 1, values for $Q^{*}$ and $Q^{* \prime}$ for $T=580 \mathrm{~K}, T=780 \mathrm{~K}$, and $T=1280 \mathrm{~K}$ are computed. By exploring the temperature dependence, some fundamental insight is obtained. In particular, $Q^{*}$ is shown to gradually increase with increasing temperature, whereas the reduced heat of transport $Q^{* \prime}=Q^{*}-h_{2}$, where $h_{2}$ is the partial enthalpy of hydrogen, is found to be essentially temperature independent. Moreover, by analyzing in detail different contributions to $Q^{*}$, several important features emerge. Specifically, hydrogen atoms that are in the process of diffusing are found to have higher energy in comparison the average energy of a hydrogen atom. The difference between the energy of a diffusing hydrogen atom and the average energy of a hydrogen atom is found to be comparable to the energy of the migration barrier, which has previously been suggested by simple theoretical arguments. ${ }^{4}$ However, the virial term in the energy flux is found to be less than what would be expected based on the average contribution of the hydrogen atoms to the stress tensor. For the first time, it is shown how significant positive contributions to $Q^{*}$ due to the excited state of the hopping can nevertheless be consistent with a negative value of the reduced heat of transport $Q^{* \prime}$. The analysis includes a calculation of the local stress component along the hopping direction, which is shown to exhibit a minimum when the hydrogen atom is at the transition state. These observations provide important insight that can be applied to other materials systems, especially where the Green-Kubo approach is not practical due to slow diffusion rates.

\section{SIMULATION APPROACH AND RESULTS}

The details of the simulations reported here were previously given in Ref. 1. In short, the empirical pair potentials for the description of the Pd:H system were taken from Ref. 2. As previously described, ${ }^{1}$ the potentials from Ref. 2 were smoothed at the cutoff distance $r_{c}$ using a standard approach. The objective was to remove discontinuities primarily seen in the $\mathrm{Pd}-\mathrm{H}$ and $\mathrm{H}-\mathrm{H}$ interactions. Specifically, the potentials were smoothed using the expression,

$$
V_{\alpha \beta}(r)=V_{\alpha \beta}^{(0)}(r)-V_{\alpha \beta}^{(0)}\left(r=r_{c}\right)-\left(r-r_{c}\right)\left[\frac{d V_{\alpha \beta}^{(0)}}{d r}\right]_{r=r_{c}},
$$


where $V_{\alpha \beta}(r)$ is the smoothed potential, and $V_{\alpha \beta}^{(0)}$ is the original Gillan potential. The subscript $\alpha \beta$ refers to the particular pair, $\mathrm{Pd}-\mathrm{Pd}, \mathrm{Pd}-\mathrm{H}$, or H-H. Although not discussed in Ref. 1, the general behavior of the potential was tested to be certain the description of the $\mathrm{Pd}-\mathrm{H}$ system remained reasonable. In particular, the phonon modes for pure Pd were computed and found to be shifted by at most $\sim 2 \%$. In the original paper by Gillan, the Pd-Pd interactions were adjusted to accurately reproduce the experimental phonon spectra. ${ }^{2}$ Next, the local vibrational frequency of hydrogen atoms was computed with the assumption that the $\mathrm{Pd}$ atoms remain fixed. In particular, we follow Gillan ${ }^{2}$ and compute

$$
m \omega_{0}^{2}=2\left[\frac{d^{2} V_{P d H}}{d r^{2}}+\frac{2}{r} \frac{d V_{P d H}}{d r}\right]_{r=a_{0} / 2} .
$$

Here, $m$ is the hydrogen mass and $\omega_{0} / 2 \pi$ is the local vibrational frequency of a hydrogen atom at an octahedral interstitial site. The term in brackets is evaluated at the nearest neighbor bond length $a_{0} / 2$. Gillan used the value $\omega_{0} / 2 \pi$ $=15.9 \mathrm{THz}$ found in experiment to fix the parametrization of the $\mathrm{Pd}-\mathrm{H}$ interaction. ${ }^{2}$ In fact, for the parameterization used by Gillan, we compute $\omega_{0} / 2 \pi=16.12 \mathrm{THz}$, which is slightly larger than the experimental value. For the smoothed potential, we compute $\omega_{0} / 2 \pi=16.34 \mathrm{THz}$. This value is still in reasonably good agreement with experiment. For harmonic properties, the reason why the smoothing procedure does not have a significant effect is quite clear. Specifically, Eq. (1) above does not result in a change in the second derivative $d^{2} V_{\alpha \beta} / d r^{2}$, which is the most important for determining the harmonic properties. The primary effect is a constant energy shift and a small shift in forces which results in only minor changes in the harmonic properties.

Further, in Ref. 1, the activation energy for hopping was determined and found to be in good agreement with the value reported by Gillan. ${ }^{2}$ The activation energy is perhaps more important for transport than the harmonic properties. Specifically, the value found in Ref. 1 using the smoothed potential was $\Delta U_{a c t}=0.25 \mathrm{eV}$ which is very reasonable compared to the value $\Delta U_{a c t}=0.29 \mathrm{eV}$ reported by Gillan, and apparently in better agreement with the experimental value $\Delta U_{a c t}=0.23 \mathrm{eV}^{2}$ In short, based on both harmonic frequencies and energies most relevant for diffusion, the smoothing procedure only slightly changes the results and removes undesirable discontinuities found with the original Gillan potential.

For Green Kubo calculations reported here and previously in Ref. 1, we use a cubic simulation supercell with $N_{1}=108 \mathrm{Pd}$ atoms and $N_{2}=20$ hydrogen atoms. Each simulation was performed at fixed volume corresponding to the fcc lattice parameter $a_{0}=4.07 \AA$ independent of temperature.

At each step in the MD simulation, the energy and mass fluxes were computed in a center-of-mass reference frame. The mass flux for hydrogen is computed at each step using the expression,

$$
\vec{J}_{2}=\sum_{i=1}^{N_{2}} \vec{v}_{i}
$$

where the summation includes only the hydrogen atoms each with velocity vector $\vec{v}_{i}$. The energy flux is computed by ${ }^{5}$

$$
\vec{J}_{q}=\sum_{i} \vec{v}_{i} \epsilon_{i}+\frac{1}{2} \sum_{i<j} \vec{r}_{i j}\left[\vec{F}_{i j} \cdot\left(\vec{v}_{i}+\vec{v}_{j}\right)\right],
$$

where the summations are over all particles, $\mathrm{Pd}$ and $\mathrm{H}$, and $\vec{F}_{i j}$ is the force on atom $i$ due to its interaction with atom $j$. The partial energy $\epsilon_{i}$ is given by

$$
\epsilon_{i}=\frac{1}{2} m_{i} \vec{v}_{i} \cdot \vec{v}_{i}+\frac{1}{2} \sum_{j \neq i} V_{i j}\left(r_{i j}\right),
$$

where $V_{i j}$ is the pair-potential interaction between particles $i$ and $j$ separated by a distance $r_{i j}$.

The objective of the MD simulations is to compute the transport coefficients $L_{22}, L_{2 q}, L_{q 2}$, and $L_{q q}$ which relate the thermodynamic driving forces to the mass and heat fluxes by ${ }^{6}$

$$
\begin{aligned}
& \vec{J}_{2}=\frac{\Omega}{k_{B} T}\left[L_{22} \vec{X}_{2}+L_{2 q} \vec{X}_{q}\right], \\
& \vec{J}_{q}=\frac{\Omega}{k_{B} T}\left[L_{q 2} \vec{X}_{2}+L_{q q} \vec{X}_{q}\right],
\end{aligned}
$$

where $\Omega$ is the system volume. The driving forces are defined by

$$
\begin{gathered}
\vec{X}_{2}=-T \vec{\nabla}\left(\frac{\mu_{2}}{T}\right), \\
\vec{X}_{q}=-\frac{1}{T} \vec{\nabla} T,
\end{gathered}
$$

where $\mu_{2}$ is the chemical potential of hydrogen.

The Green-Kubo method for calculation of the transport parameters uses the integrated time-correlation functions, ${ }^{3,7,8}$

$$
\begin{aligned}
& \zeta_{22}(\tau)=\frac{1}{3 \Omega} \int_{0}^{\tau}\left\langle\vec{J}_{2}(\tau) \cdot \vec{J}_{2}(0)\right\rangle d \tau, \\
& \zeta_{2 q}(\tau)=\frac{1}{3 \Omega} \int_{0}^{\tau}\left\langle\vec{J}_{2}(\tau) \cdot \vec{J}_{q}(0)\right\rangle d \tau .
\end{aligned}
$$

The relevant transport parameters are found by taking the long-time limit of the integrated time-correlation functions,

$$
\begin{aligned}
& L_{22}=\lim _{\tau \rightarrow \infty} \zeta_{22}(\tau), \\
& L_{2 q}=\lim _{\tau \rightarrow \infty} \zeta_{2 q}(\tau) .
\end{aligned}
$$

Finally, the value of the heat of transport $Q^{*}$ for hydrogen is found from

$$
Q^{*}=\frac{L_{2 q}}{L_{22}}
$$

Simulations were performed at $T=580 \mathrm{~K}, T=780 \mathrm{~K}$, and $T=1280 \mathrm{~K}$. The results in Ref. 1 for $T=980 \mathrm{~K}$ are also included here. To obtain well-converged transport 


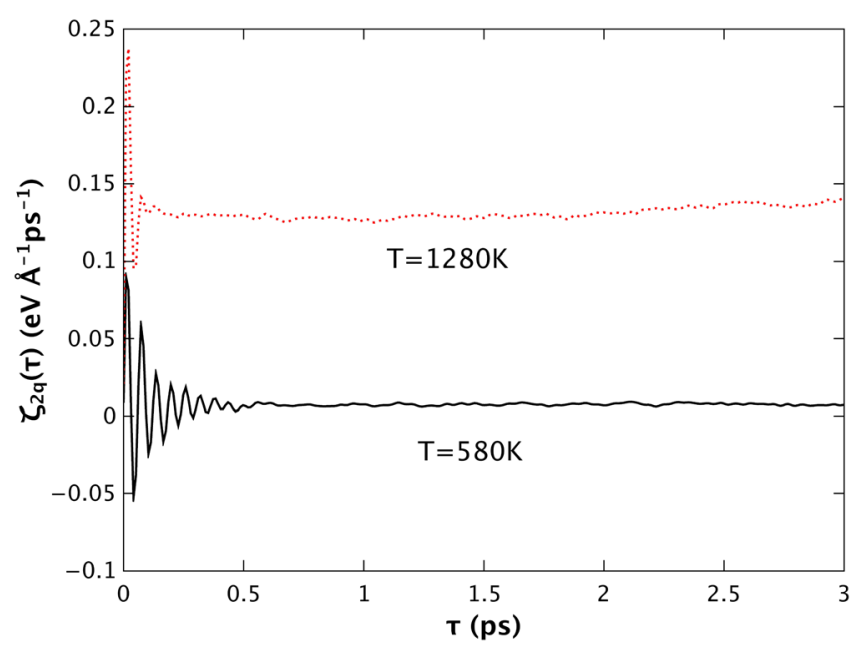

FIG. 1. The integrated correlation function $\zeta_{2 q}(\tau)$ as a function of integration time $\tau$ for $T=580 \mathrm{~K}$ (black solid line) and $T=1280 \mathrm{~K}$ (red dotted line).

parameters, a large number of independent MD simulations were performed. In particular, for $T=780 \mathrm{~K}, T=980 \mathrm{~K}$, and $T=1280 \mathrm{~K}, 360$ independent simulations were performed. Each of these independent MD simulations included $2.1 \times 10^{5} \mathrm{MD}$ steps and a total simulation time of $87 \mathrm{ps}$. The first 4.14 ps of these simulations was used for equilibration at constant volume and temperature and the trajectories were not used in the Green-Kubo calculations. After the initial equilibration period, the heat and mass fluxes were computed for constant volume and energy. For the case $T=580 \mathrm{~K}$, we found that due to a reduced hopping rate of the hydrogen atoms, a longer simulation time was required. To obtain accurate results at $T=580 \mathrm{~K}$, there were still 360 independent MD simulations used to compute the transport parameters. However, for $T=580 \mathrm{~K}$, each simulation was run for a total of $667 \mathrm{ps}$. Altogether, the time-correlation functions were averaged using $29.8 \mathrm{~ns}$ of simulation time for $T=780 \mathrm{~K}, T=980 \mathrm{~K}$, and $T=1280 \mathrm{~K}$. For $T=580 \mathrm{~K}$, a total time of $238.7 \mathrm{~ns}$ of data was used.

In Fig. 1, the integrated time-correlation function $\zeta_{2 q}(\tau)$ is shown as a function of integration time $\tau$ for simulation with $T=580 \mathrm{~K}$ and $T=1280 \mathrm{~K}$. In Fig. $2, \zeta_{22}(\tau)$ is shown for the same two temperatures. It is clear that both functions converge to a positive value within about $\sim 1-2$ ps. Beyond $\sim 2$ ps, the correlation functions $\left\langle\vec{J}_{2}(\tau) \cdot \vec{J}_{q}(0)\right\rangle$ and $\left\langle\vec{J}_{2}(\tau) \cdot \vec{J}_{2}(0)\right\rangle$ are essentially zero and do not contribute to the integral. In Table I, we show the computed values of $L_{22}$, $L_{2 q}$, and the heat of transport $Q^{*}$ for each temperature simulated. The values used for $L_{2 q}$ and $L_{22}$ are obtained from $\zeta_{2 q}(\tau)$ and $\zeta_{22}(\tau)$ using the integration time $\tau=2.0 \mathrm{ps}$. As in

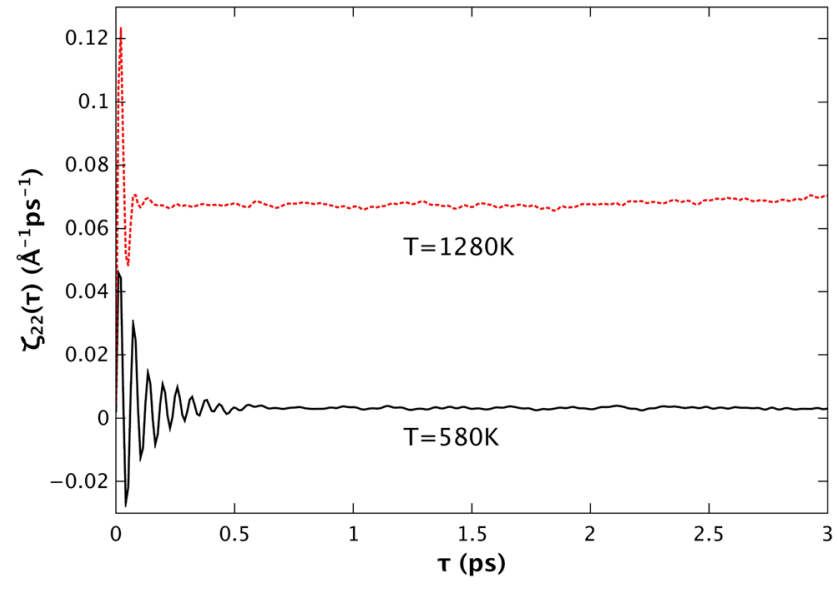

FIG. 2. The integrated correlation function $\zeta_{22}(\tau)$ as a function of integration time $\tau$ for $T=580 \mathrm{~K}$ (black solid line) and $T=1280 \mathrm{~K}$ (red dotted line).

Ref. 1, integration to longer times $\tau$ yields no systematic change but increased statistical error. Table I includes the estimated statistical error in the reported values of $L_{2 q}$ and $L_{22}$ determined from an analysis of smaller data sets. The values for the heat of transport $Q^{*}=L_{2 q} / L_{22}$ are also shown in Table I. Finally, values for the partial enthalpy of hydrogen $h_{2}$ and $Q^{* \prime}=Q^{*}-h_{2}$ are also shown in Table I. The value of $h_{2}$ for each temperature is determined from a constant volume and temperature calculation with $10^{5} \mathrm{MD}$ steps, with the first $10^{4}$ steps not included in the averaging. The expression for $h_{2}$ is given in Ref. 1 and also below. We also discuss below in more detail the expression for $h_{2}$. In particular, we include a brief discussion of the derivation of a microscopic expression for $h_{2}$ in multicomponent mixtures from Refs. 9-11. Specifically, we contrast $h_{2}$ computed using the microscopic expression valid for computing $Q_{2}^{* \prime}$, with the standard thermodynamic expression for $h_{2}$ used by Gillan. ${ }^{3}$ In addition, we compare values of $h_{2}$ computed in both the center-of-mass reference frame, and in a frame moving with the average velocity of the hydrogen atoms, and show that the resulting values are nearly equal.

The activated nature of the diffusion process can be seen from an Arrhenius plot of the computed values of $L_{22}$, as shown in Fig. 3. Also in Fig. 3 the computed values of $L_{2 q}$ are also shown to exhibit activated behavior. This is not surprising, since the time-correlation function used to compute $L_{2 q}$ is only nonzero in the event that significant hydrogen diffusion takes place. The activation energy for both $L_{2 q}$ and $L_{22}$ obtained from Fig. 3 is in the range $0.27-0.29 \mathrm{eV}$, which is larger than the value $0.25 \mathrm{eV}$ reported previously. ${ }^{1}$ The origin for this difference is unclear. However, it may be due to

TABLE I. Values of the transport parameters $L_{2 q}$ and $L_{22}$ obtained from Green-Kubo calculations. Also included are the energies $Q^{*}, h_{2}$, and $Q^{* \prime}=Q^{*}-h_{2}$ described in the text.

\begin{tabular}{lcccc}
\hline \hline$T(\mathrm{~K})$ & $L_{2 q}\left(\mathrm{eV} \mathrm{ps}^{-1} \AA^{-1}\right)$ & $L_{22}\left(\mathrm{ps}^{-1} \AA^{-1}\right)$ & $Q^{*}(\mathrm{eV})$ & $Q^{* \prime}(\mathrm{eV})$ \\
\hline 580 & $0.00567 \pm 0.00001$ & $0.00326 \pm 0.00001$ & $1.738 \pm 0.01$ & 1.946 \\
780 & $0.0249 \pm 0.001$ & $0.0138 \pm 0.0004$ & $1.803 \pm 0.12$ & 1.989 \\
980 & $0.0620 \pm 0.001$ & $0.0339 \pm 0.003$ & $1.833 \pm 0.04$ & 2.038 \\
1280 & $0.1294 \pm 0.005$ & $0.0671 \pm 0.003$ & $1.927 \pm 0.07$ & -0.01 \\
\hline \hline
\end{tabular}




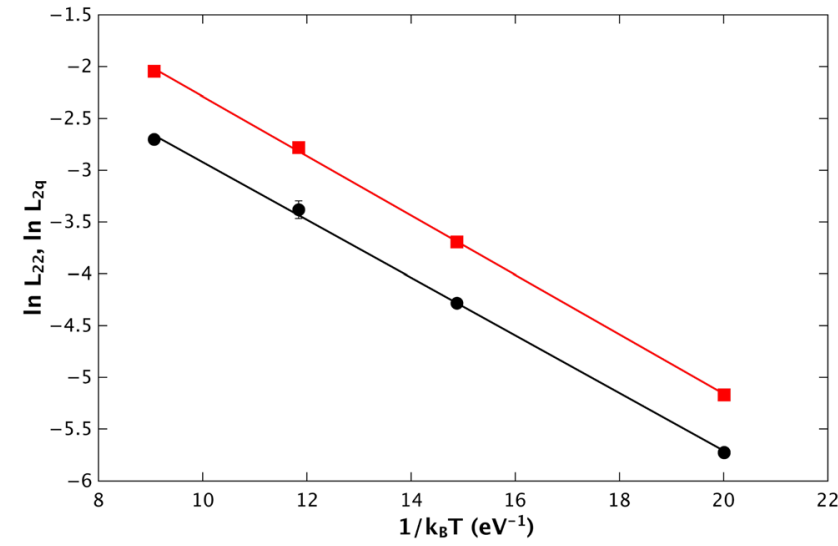

FIG. 3. Arrhenius plot for $L_{22}$ (black circles) and $L_{2 q}$ (red squares) including linear fits. For $L_{22}$, the slope corresponds to an energy $-0.28 \mathrm{eV}$, while for $L_{2 q}$, the fit slope results in an energy $-0.29 \mathrm{eV}$. Error bars are added for each point, although in most cases the error bars are comparable to or smaller than the points.

correlated hopping especially at higher temperatures. Alternately, it could be that hydrogen-hydrogen interactions limit multiple correlated hops that might otherwise occur especially at higher temperatures, thereby resulting in higher apparent values of the activation energy.

While the activated behavior of $L_{2 q}$ and $L_{22}$ effectively cancels in the value of $Q^{*}=L_{2 q} / L_{22}$, the results in Table I show quite clearly that $Q^{*}$ increases weakly with increasing system temperature $T$. Assuming linear dependence of $Q^{*}$ on temperature, the computed data can be fit by $Q_{(f i t)}^{*}(T)=1.59 \mathrm{eV}+3.00 k_{B} T$. The physical interpretation of $Q^{*}$ is that it represents the energy transported by the diffusing species. The linear dependence of $Q^{*}$ on temperature shows that as $T$ increases, the energy carried by the diffusing species increases. By contrast, to within the statistical error, $Q^{* \prime}$ appears to be essentially temperature independent.

To gain further insight into the value of $Q^{*}$, we have also computed separate contributions to the transport coefficients. In particular, we define separate contributions to the energy flux due to the kinetic energy, potential energy, and virial terms by

$$
\begin{gathered}
\vec{J}_{q}^{(k i n)}=\sum_{i} \vec{v}_{i}\left[\frac{1}{2} m_{i} \vec{v}_{i} \cdot \vec{v}_{i}\right], \\
\vec{J}_{q}^{(\text {pot })}=\frac{1}{2} \sum_{i} \vec{v}_{i}\left[\sum_{j \neq i} V_{i j}\left(r_{i j}\right)\right], \\
\vec{J}_{q}^{(v i r)}=\frac{1}{2} \sum_{i<j} \vec{r}_{i j}\left[\vec{F}_{i j} \cdot\left(\vec{v}_{i}+\vec{v}_{j}\right)\right] .
\end{gathered}
$$

Obviously, the individual contributions sum to give the net energy flux,

$$
\vec{J}_{q}=\vec{J}_{q}^{(k i n)}+\vec{J}_{q}^{(\text {pot })}+\vec{J}_{q}^{(v i r)}
$$

Using these separate contributions to the energy flux $\vec{J}_{q}$, we next define the integrated time-correlation functions,

$$
\begin{aligned}
& \zeta_{2 q}^{(k i n)}(\tau)=\frac{1}{3 \Omega} \int_{0}^{\tau}\left\langle\vec{J}_{2}(\tau) \cdot \vec{J}_{q}^{(k i n)}(0)\right\rangle d \tau, \\
& \zeta_{2 q}^{(p o t)}(\tau)=\frac{1}{3 \Omega} \int_{0}^{\tau}\left\langle\vec{J}_{2}(\tau) \cdot \vec{J}_{q}^{(\text {pot })}(0)\right\rangle d \tau, \\
& \zeta_{2 q}^{(v i r)}(\tau)=\frac{1}{3 \Omega} \int_{0}^{\tau}\left\langle\vec{J}_{2}(\tau) \cdot \vec{J}_{q}^{(\text {vir })}(0)\right\rangle d \tau .
\end{aligned}
$$

From these expressions, the contributions to $L_{2 q}$ due to the kinetic, potential, and virial terms can be determined for long integration time $\tau$,

$$
\begin{aligned}
& L_{2 q}^{(k i n)}=\lim _{\tau \rightarrow \infty} \zeta_{2 q}^{(k i n)}(\tau), \\
& L_{2 q}^{(p o t)}=\lim _{\tau \rightarrow \infty} \zeta_{2 q}^{(p o t)}(\tau), \\
& L_{2 q}^{(v i r)}=\lim _{\tau \rightarrow \infty} \zeta_{2 q}^{(v i r)}(\tau) .
\end{aligned}
$$

Finally, the contributions to the total heat of transport $Q^{*}$ are defined by

$$
\begin{aligned}
& Q^{*(k i n)}=\frac{L_{2 q}^{(k i n)}}{L_{22}}, \\
& Q^{*(p o t)}=\frac{L_{2 q}^{(p o t)}}{L_{22}}, \\
& Q^{*(v i r)}=\frac{L_{2 q}^{(v i r)}}{L_{22}} .
\end{aligned}
$$

\begin{tabular}{|c|c|c|c|c|c|c|c|c|c|}
\hline$T(\mathrm{~K})$ & $Q^{*(k i n)}$ & $Q^{*(p o t)}$ & $Q^{*(v i r)}$ & $u_{2}$ & $p_{2} \Omega$ & $Q^{*(k i n)}-K$ & $Q^{*(p o t)}-u_{2}+K$ & $Q^{*(k i n)}+Q^{*(p o t)}-u_{2}$ & $Q^{*(v i r)}-p_{2} \Omega$ \\
\hline 580 & 0.198 & 0.761 & 0.779 & 0.773 & 1.173 & 0.123 & 0.063 & 0.186 & -0.394 \\
\hline 780 & 0.231 & 0.761 & 0.811 & 0.810 & 1.179 & 0.130 & 0.052 & 0.182 & -0.368 \\
\hline 980 & 0.288 & 0.763 & 0.782 & 0.847 & 1.191 & 0.161 & 0.043 & 0.204 & -0.409 \\
\hline 1280 & 0.331 & 0.764 & 0.832 & 0.894 & 1.225 & 0.166 & 0.035 & 0.201 & -0.393 \\
\hline
\end{tabular}

The results for $Q^{*(k i n)}, Q^{*(p o t)}$, and $Q^{*(v i r)}$ are summarized in Table II. The values of $Q^{*(k i n)}$ and $Q^{*(p o t)}$ were in each case determined from a smaller number of simulations where the relevant data was saved. For each case, 40 independent simulations with $2.0 \times 10^{5} \mathrm{MD}$ steps were used to compute $Q^{*(\mathrm{kin})}$ and $Q^{*(\text { pot })}$. The value of $Q^{*(\mathrm{vir})}$, which general shows more statistical error than the other contributions, was determined by subtracting $Q^{*(k i n)}$ and $Q^{*(p o t)}$ from the values of $Q^{*}$ determined from the complete simulation dataset. For each temperature, $Q^{(k i n)}$ is greater than the average

TABLE II. Computed energies in eV for the thermodiffusion of hydrogen. 
kinetic energy $K=(3 / 2) k_{B} T$, indicating that the hydrogen atoms are in an excited state during hopping. The average potential energy of a hydrogen atom is found by subtracting the average kinetic energy $K$ from the average energy of a hydrogen $u_{2}$. As in Ref. 1, the average energy of a hydrogen atom is computed from

$$
u_{2}=\frac{1}{N_{2}} \sum_{i=1}^{N_{2}}\left\langle\epsilon_{i}\right\rangle
$$

where $\epsilon_{i}$ is the local energy of hydrogen atom $i$ defined from Eq. (5). From Table II, it is seen that $Q^{*(p o t)}-\left(u_{2}-K\right)$ is positive, which is again consistent with the excited state of the hydrogen atoms during the hopping process. Interestingly, from Table II, it can be seen that the excess kinetic of the hydrogen atoms during hopping increases with increasing temperature $T$, whereas the excess potential energy decreases with increasing temperature. Finally, the sum of the excess kinetic and potential energy, given by $Q^{*(k i n)}+Q^{*(p o t)}-u_{2}$, also given in Table II, is relatively temperature independent with a magnitude comparable, although a bit smaller, than the migration barrier $\sim 0.25 \mathrm{eV}$.

While comparing $Q^{*(k i n)}+Q^{*(p o t)}$ with the average hydrogen energy $u_{2}$ provides important insight, complete understanding of thermodiffusion emerges by also comparing $Q^{*(v i r)}$ with $p_{2} \Omega$, where $p_{2}$ is the average partial pressure of hydrogen. This term occurs in the definition of the partial enthalpy of hydrogen $h_{2}=u_{2}+p_{2} \Omega$. Specifically, we consider here the difference $Q^{*(v i r)}-p_{2} \Omega$. From Table II, this quantity is negative for each temperature studied. This term is responsible for the negative value of $Q^{* \prime}$.

It is possible to obtain insight into why $Q^{*(v i r)}-p_{2} \Omega$ is negative. From Ref. 1, the contribution a single hydrogen atom $i$ to the element $\pi_{\mu \nu}$ of the stress tensor is defined by

$$
\pi_{\mu \nu, i} \Omega=\left[m_{2}\left\langle v_{i, \mu} v_{i, \nu}\right\rangle+\frac{1}{2} \sum_{j \neq i}\left\langle F_{i j, \mu} r_{i j, \nu}\right\rangle\right]
$$

where the angle brackets refer to an average over an equilibrium ensemble of states. The relationship between $p_{2} \Omega$ and the contributions to the stress tensor due to the $N_{2}$ hydrogen atoms is

$$
p_{2} \Omega=\frac{1}{3 N_{2}} \sum_{i=1}^{N_{2}}\left(\sum_{\mu=1}^{3} \pi_{\mu \mu, i}\right)
$$

We next consider how to analyze key contributions to $Q^{*(v i r)}$. Consider a single hydrogen atom hopping along the $\mathrm{z}$ direction $(\mu=3)$, and assume that the hydrogen atom is isolated from other hydrogen atoms so that it interacts only with the Pd lattice. Then, considering only the direct contribution to the virial flux along the direction of the hop $(\mu=3)$ explicitly due to the displacement of the hopping atom, we define

$$
J_{3}^{(v i r) \prime}=\frac{1}{2} \sum_{j \neq i} F_{i j, 3} r_{i j, 3} v_{i, 3}
$$

Clearly, $J_{3}^{(v i r) \prime}$ is only part of the complete virial energy flux defined in Eq. (17). We define $Q_{h}^{*(v i r)}$ by an integration of Eq. (31) is integrated over an ensemble of successful hops,

$$
Q_{h}^{*(v i r)}=\frac{1}{2 a} \sum_{j \neq i} \int_{r_{i, 3}=0}^{r_{i, 3}=a}\left\langle F_{i j, 3} r_{i j, 3}\right\rangle_{h} d r_{i, 3},
$$

where the integral is over the path of the hydrogen atom as it hops from $r_{i, 3}=0$ to $r_{i, 3}=a$. The length $a$ is the separation between lattice sites along the [110] hopping direction, hence $a=2.88 \AA$. The angle brackets represent an ensemble average only over successful hops of the hydrogen atom $i$. It is important to note that Eq. (32) doe not account for possibly important contributions to $J^{*(v i r)}$ that correspond to conductive energy transport. This will discussed more fully below. Nevertheless, the close relationship between the element of the stress tensor $\pi_{33, i}$ in Eq. (29) and the term in the integrand in Eq. (32) is immediately evident, and it is possible to obtain important insight into the negative value of $Q^{*(v i r)}-p_{2} \Omega$ by considering the difference $Q_{h}^{*(v i r)}-p_{2} \Omega$.

In the following, we show that it is possible to establish trends in $Q^{*(v i r)}$, and obtain insight, without exactly evaluating Eq. (32). Instead of computing an ensemble average over successful hops, we instead constrained the hopping hydrogen atom at several points along the transition path following the method described in Ref. 1 and performed an equilibrium MD calculation at each point for $T=980 \mathrm{~K}$. In particular, the motion of the hydrogen atom is constrained along the [110] crystallographic direction, while remaining free to move in the two perpendicular dimensions. Hence, for each simulation, the coordinate $r_{i, 3}$ is fixed at some constant value throughout the simulation. The simulation cell contained $2688 \mathrm{Pd}$ atoms and a single hydrogen atom, and for each constrained coordinate the calculation integrated $10^{5} \mathrm{MD}$ steps. At each constrained coordinate of the hydrogen atom, we computed the average contribution to the stress tensor due to the added hydrogen atom, with the first $10^{4} \mathrm{MD}$ steps of equilibration not included in the averaging. Because the hydrogen atom is constrained, the component of its velocity $v_{i, 3}$ along the hopping direction is always zero. Then we define $Q_{c}^{*(v i r)}$,

$$
\begin{aligned}
Q_{c}^{*(v i r)} & =\frac{1}{2 a} \sum_{j \neq i} \int_{r_{i, 3}=0}^{r_{i, 3}=a}\left\langle F_{i j, 3} r_{i j, 3}\right\rangle_{(c)} d r_{i, 3} \\
& =\frac{1}{a} \int_{r_{i, 3}=0}^{r_{i, 3}=a} \pi_{33, i}\left(r_{i, 3}\right) \Omega d r_{i, 3} .
\end{aligned}
$$

This is very closely related to the quantity $Q_{h}^{*(v i r)}$ in Eq. (32), with the nevertheless important difference that the ensemble in Eq. (32) is over successful hops, whereas the ensemble in Eq. (33) is over an equilibrium ensemble of states with the hydrogen atom constrained at some coordinate along its hopping trajectory. Hence, we expect that $Q_{h}^{*(v i r)}$ and $Q_{c}^{*(v i r)}$ are not equal. Rather, $Q_{c}^{*(v i r)}$, which can be readily computed, is an approximation to the quantity $Q_{h}^{*(v i r)}$, and hence can provide insight into $Q^{*(v i r)}$.

In Fig. 4 , the quantity $\pi_{33, i}\left(r_{i, 3}\right) \Omega$ plotted as a function of the coordinate $r_{i, 3}$ of the hydrogen atom along the hopping 


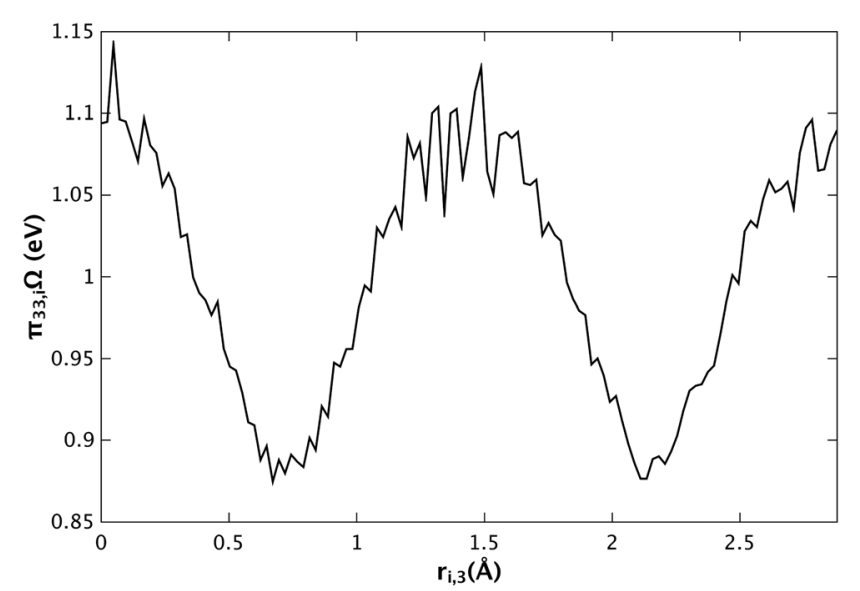

FIG. 4. Stress energy $\pi_{33, i} \Omega$ associated with the hydrogen atom $i$ as it is displaced along the hopping path by constraining the coordinate $r_{3, i}$. At the transition state, $\pi_{33, i} \Omega$ exhibits a minimum, whereas at the octahedral interstitial sites $\pi_{33, i} \Omega$ is maximum.

trajectory. The path of the hydrogen atom allows it to explore three different octahedral interstitial sites at $r_{i, 3}=0$, $r_{i, 3}=1.44 \AA$, and $r_{i, 3}=2.88 \AA$. The two transition states, therefore, occur at $r_{i, 3}=0.72 \AA$ and $r_{i, 3}=2.16 \AA$. The important points to note are that $\pi_{33, i}\left(r_{i, 3}\right) \Omega$ is maximum and comparable to $p_{2} \Omega$ when the hydrogen atom is near an interstitial site, and minimum near the two transition states. The integral in Eq. (33) results in $Q_{c}^{*(v i r)}=1.00 \pm 0.02 \mathrm{eV}$. This is greater than $Q^{*(v i r)}=0.782 \mathrm{eV}$ from the Green-Kubo results for $T=980 \mathrm{~K}$ in Table II. However, $Q_{c}^{*(v i r)}$ is less than the value $p_{2} \Omega=1.191 \mathrm{eV}$ using the data from Table II. For $T=980 \mathrm{~K}$, we then find that $Q_{c}^{*(\text { vir })}-p_{2} \Omega=-0.191 \mathrm{eV}$. The negative value of this quantity, which occurs due to the reduced average stress component $\pi_{33, i}\left(r_{i, 3}\right)$ as the hydrogen is displaced along its hopping trajectory, is consistent with the negative value of $Q^{*(v i r)}-p_{2} \Omega$ seen in Table II. This analysis partly elucidates the negative value for reduced heat of transport $Q^{* \prime}$ predicted by the Green-Kubo results.

It is possible to develop at least a qualitative argument to explain why $\pi_{33}\left(r_{i, 3}\right)$ should be minimum when $r_{i, 3}$ corresponds to the transition state. Generally, when atoms diffuse in solids, the transition state corresponds to the atom passing through "gate" atoms which allow only a narrow space for the diffusing species to pass through. At the transition state, the diffusing species is closest to the gate atoms, and generally the forces between the gate atoms and the hopping species are perpendicular to hop direction. It is reasonable then to expect that the stress component along the hopping direction, for example $\pi_{33, i}$ at the transition state should be minimum, consistent with what is shown in Fig. 4. It then stands to follow that the stress components perpendicular to the hopping direction should be maximum at the transition state. In Fig. 5, the components $\pi_{11}\left(r_{i, 3}\right) \Omega$ and $\pi_{22}\left(r_{i, 3}\right) \Omega$ are shown as a function of the coordinate $r_{i, 3}$. Here, the Cartesian component $\mu=1$ corresponds to the $[\overline{1} 10]$ direction in the fcc lattice, and $\mu=2$ corresponds to the [001] direction. The values of $\pi_{11}\left(r_{i, 3}\right) \Omega$ show a clear maximum at the transition state, consistent with this qualitative picture. By contrast, the data for $\pi_{22}\left(r_{i, 3}\right) \Omega$ are quite noisy, but shows much less variation along the transition path. However, it

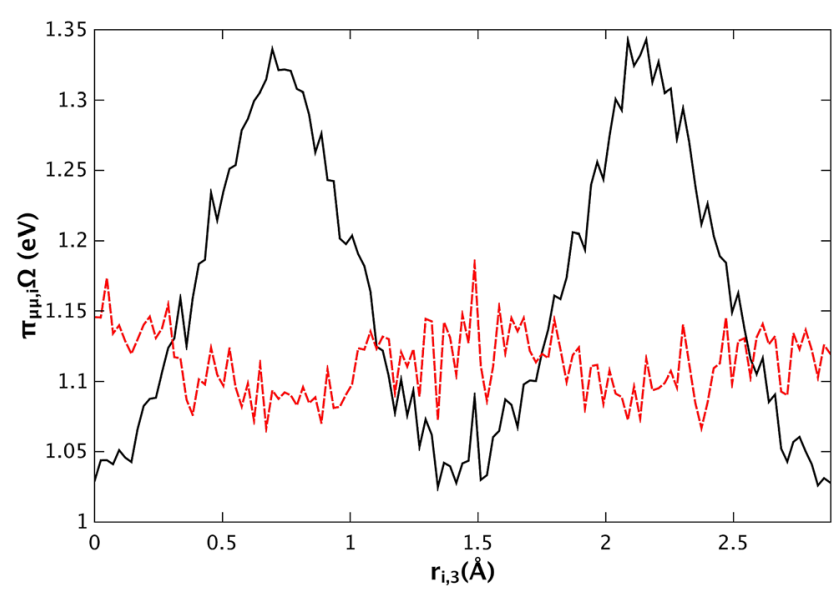

FIG. 5. Energy terms $\pi_{11, i} \Omega$ (solid black line) and $\pi_{22, i} \Omega$ (dashed red line) plotted as a function of the hydrogen coordinate $r_{3, i}$ along the hopping path. For $\pi_{11, i} \Omega$, a very strong maximum is observed when the hydrogen is held at the transition state.

seems likely that there is actually a weak minimum for $\pi_{22}\left(r_{i, 3}\right) \Omega$ near the transition state. However, the average pressure $p=\frac{1}{3} \sum_{\mu=1}^{3} \pi_{\mu \mu, i}$ varies only weakly with $r_{i, 3}$, consistent with the picture that, at the transition state, the stress along the hopping direction is minimum while the overall stress in the perpendicular directions is maximum.

Next, it is important to comment on the differences between $Q_{c}^{*(v i r)}$ and $Q^{*(v i r)}$ obtained from the Green-Kubo calculations. Perhaps the most important distinction is that $Q_{c}^{*}(v i r)$ was obtained from an equilibrium ensemble of states with the hydrogen atom constrained along the hopping path, while the value of $Q^{*(v i r)}$ obtained from Green Kubo results from an ensemble of successful hopping trajectories as is the quantity $Q_{h}^{*}$ defined in Eq. (32). Thus, the prediction in Eq. (32) corresponds to a different ensemble of states than that used in Eq. (33). It is possible to speculate on the differences. First, diffusion occurs when the diffusing species has enough energy to overcome the migration barrier. However, the barrier itself is not static, but rather fluctuating. In Ref. 1, it was shown that the activation free energy was significantly lower than the activation energy, corresponding to an activation entropy of about $1.54 k_{B}$. Hence, when lattice fluctuations occur so that $\pi_{33, i} \Omega$ is decreased, hopping should be more likely to occur. By contrast, if lattice fluctuations result in an increase in $\pi_{33, i} \Omega$, successful hops will be less likely to occur. In the evaluation of Eq. (33), no consideration was given to the likelihood of a particular configuration in the ensemble corresponding to a successful hop. Hence, if the ensemble of successful hops tends to correlate with lower values of $\pi_{33, i} \Omega, Q_{h}^{(v i r) *}$ from Eq. (32) should predict a smaller value in comparison to the prediction $Q_{c}^{(v i r)}$ from Eq. (33). We then expect that $Q_{h}^{(v i r) *}$ would be a more complete prediction for $Q^{(v i r) *}$ from the Green Kubo results, but we do not know how $Q_{h}^{(v i r) *}$ might be evaluated.

We note that in defining the flux $J_{3}^{(v i r) \prime}$, several terms present in the definition for $\vec{J}^{(v i r)}$ were not considered. The terms that were neglected were those that do not explicitly depend on the velocity vector $\vec{v}_{i}$ of the hopping hydrogen atom. Nevertheless, these terms play some role which is likely also important. Primarily, the other terms should be 
responsible for heat conduction since apart from the hydrogen atom which is diffusing, the Pd atoms simply execute oscillations about their respective lattice sites. In the future, it would be interesting and important to try and separately analyze the explicitly conductive and convective parts of $\vec{J}^{(v i r)}$. It is possible that conductive heat transport might make either positive or negative contributions to $Q^{*(v i r)}$. However, the analysis above still clearly shows the some of the key contributions that lead to a negative value for $Q^{*(v i r)}-p_{2} \Omega$ and hence negative values of $Q^{* \prime}=Q^{*}-p_{2} \Omega$.

Finite-size effects were also explored, and found to be relatively unimportant. It might be thought that finite-size effects can emerge due to long-range heat conduction. Specifically, we simulated a larger cubic simulation cell with $N_{1}=2916$ and $N_{2}=540$. In both cases, the procedure was the same as described above, with the exception that a shorter total simulation time of $18.6 \mathrm{~ns}$ was used to compute $Q^{*}$. The larger system had the same hydrogen concentration as the $N_{1}=108$ and $N_{2}=20$ system, but with simulation dimensions three time larger than what is described above. For $T=580 \mathrm{~K}$, the computed value of $Q^{*}$ was larger by $0.05 \mathrm{eV}$. Similarly, for $T=1280 \mathrm{~K}$, the value of $Q^{*}$ was increased by $0.04 \mathrm{eV}$ in comparison to the results found with the smaller cell. In both cases, the increase was due to the virial energy flux, suggesting a role for lattice heat conduction. It may be that the differences are due to the fact that some heat conduction occurs over length scales larger than the simulation cell size. Alternately, in the larger system, longer wavelength vibrational modes are accessible, which may conduct heat more effectively. Nevertheless, increasing the volume of the simulation cell by a factor of 27 does not substantially change the results for $Q^{*}$. Interestingly, we find that simulation in the larger system is not more computationally intensive, since the larger system, while more intensive to integrate, also has 27 times more hydrogen hops in a given time interval.

Finally, we discuss the expression used for $h_{2}$, in contrast with the standard thermodynamic description used by Gillan in Ref. 3, specifically,

$$
h_{2}=\left(\frac{\partial H}{\partial N_{2}}\right)_{p, T, N_{1}} .
$$

In Refs. 9-11, it was found the relevant expression for $h_{2}$ for the microscopic transport theory in multicomponent systems is not given by Eq. (34). As discussed in Ref. 9, the origin of the difference is that, in deriving the expression for the energy flux from first principles, it was assumed that the potential energy is equally shared between the interacting atoms. In this way, the expression for $h_{2}$ used in this paper and in Ref. 1 emerges. As reported in Ref. 1, it was found that the thermodynamic expression in Eq. (34) and the correct microscopic definition of $h_{2}$ yield significantly different values.

There are two distinctions between the expression for $h_{2}$ used in this paper and those given in Refs. 9 and 10. First, in Refs. 9 and 10, the kinetic energy contribution to the energy flux was computed in a reference frame moving with the average velocity of the particular species. Second, rather than
TABLE III. Values of $h_{2}$ computed in the center-of-mass reference frame and also in a frame of reference moving with the average velocity of the hydrogen. For the center-of-mass reference frame, Eq. (5) is used to determine the energy of a hydrogen atom. In the frame-of-reference moving with the hydrogen, the average energy is determined from Eqs. (35)-(37). Energies are in $\mathrm{eV}$.

\begin{tabular}{lcc}
\hline \hline$T(\mathrm{~K})$ & $h_{2}$ (Eq. (5)) & $h_{2}($ Eqs. (35)-(37)) \\
\hline 580 & 1.946 & 1.927 \\
780 & 1.989 & 1.988 \\
980 & 2.038 & 2.031 \\
1280 & 2.119 & 2.112 \\
\hline \hline
\end{tabular}

using the average enthalpy $h_{2}$ above, in Refs. 9 and 10, the relevant quantities were instantaneously computed at each step of the simulation. We expect that the approach used in Refs. 9 and 10 is more general than what is presented here, but we do not expect any significant differences. In particular, we have also computed the partial energy $u_{2}$ from

$$
u_{2}=\frac{1}{N_{2}} \sum_{i=1}^{N_{2}}\left\langle\frac{1}{2} m_{2} \vec{v}_{i}^{\prime} \cdot \vec{v}_{i}^{\prime}+\frac{1}{2} \sum_{j \neq i} V_{i j}\left(r_{i j}\right)\right\rangle,
$$

where the velocities $\vec{v}_{i}^{\prime}$ are given by

$$
\vec{v}_{i}^{\prime}=\vec{v}_{i}-\vec{v}_{2},
$$

where $\vec{v}_{2}$ is the average velocity of the hydrogen atoms defined in term of the velocities $\vec{v}_{i}$ defined in the center-ofmass reference frame by

$$
\vec{v}_{2}=\frac{1}{N_{2}} \sum_{i=1}^{N_{2}} \vec{v}_{i}
$$

Then, the partial enthalpy is found from $h_{2}=u_{2}+p_{2} \Omega$. In Table III, we show values of $h_{2}$ computed in the center-ofmass frame, and also the reference frame moving with $h_{2}$ as described above. In each case, $h_{2}$ determined in the centerof-mass reference frame is slightly larger, as would be expected, but the differences are not enough to make a significant contribution to $Q^{* \prime}$. In particular, we expect that the different frames for computing $h_{2}$ lead to corrections at most $\sim 0.02 \mathrm{eV}$.

\section{SUMMARY AND CONCLUSIONS}

By separately determining the contributions $Q^{*(k i n)}$, $Q^{*(p o t)}$, and $Q^{*(v i r)}$ to the heat of transport $Q^{*}$ using GreenKubo simulations, a very clear picture of thermodiffusion has emerged. In evaluating the reduced heat of transport given by $Q^{* \prime}=Q^{*}-h_{2}$, it is of great value to consider separately $Q^{*(k i n)}+Q^{*(p o t)}-u_{2}$ and $Q^{*(v i r)}-p_{2} \Omega$. In particular, it has been shown that for hydrogen thermodiffusion in $\mathrm{Pd}$, $Q^{*(k i n)}+Q^{*(p o t)}-u_{2}$ is positive, indicating that diffusion hydrogen carries excess kinetic and potential energy. By contrast, $Q^{*(v i r)}-p_{2} \Omega$ is negative. The result is that $Q^{* \prime}$ is negative and approximately independent of temperature. This provides a fundamental insight into interstitial 
thermodiffusion; in particular, the overall sign of the reduced heat of transport depends on the balance between these positive and negative contributions to $Q^{* \prime}$.

It has often been suggested for interstitial thermodiffusion that $Q^{* \prime}$ should be comparable to the migration barrier, or at least that the migration barrier represents a limiting value for $Q^{* \prime}$. Physically, it is easy to imagine that as the result of a local fluctuation, the diffusing interstitial atom obtains enough energy to pass over the barrier, thereby transporting an amount of energy approximately equal the barrier height. The present calculations actually validate that simple picture, while demonstrating why it is incomplete. In particular, it is found that $Q^{*(\mathrm{kin})}+Q^{*(\mathrm{pot})}-u_{2}$ is comparable to the migration barrier $\sim 0.25 \mathrm{eV}$. However, this simple picture would then suggest that $Q^{* \prime}$ would always be positive, which is not the case here or in Ref. 1 .

Instead, the negative value of $Q^{* \prime}$ arises because $Q^{*(v i r)}-p_{2} \Omega$ is rather strongly negative. It is not at all straightforward to predict $Q^{*(v i r)}-p_{2} \Omega$ from simple theoretical considerations, and to date this has been neglected. Detailed calculations show that the decreased stress component along $\pi_{33, i} \Omega$ as the hydrogen atom undergoes a hop is the key feature. Fluctuations in the barrier for successful hops also should play an important role. Finally, lattice thermal conduction should also be involved, but at present that contribution is even more difficult to isolate.

Finally, it is interesting to speculate about other materials systems, based on the insight obtained here. For example, in vacancy thermodiffusion, the migration barrier for the vacancies is often thought to be a lower limit for the reduced heat of transport. However, this is not supported by experimental evidence. ${ }^{6}$ We think that insight might be obtained for this problem using the analysis reported here. Consideration of how the local stress varies along the hopping path during vacancy thermodiffusion might help explain why the migration energy does not appear to be a limiting value for the reduced heat of transport for vacancies $Q_{v}^{* \prime}$. Interestingly, the sign of the partial pressure for vacancies might not always be positive, as is the case for the hydrogen interstitials, which might suggest very different behavior. Moreover, the activation barriers are generally significantly larger for vacancy thermodiffusion, possibly resulting in a greater role for lattice heat conduction. We are currently investigating vacancy thermodiffusion in fcc metals along these lines.

\section{ACKNOWLEDGMENTS}

This work was supported by a grant from the National Science Foundation (Award No. 1106219). We would also like to acknowledge helpful correspondence from Professor Alan Lidiard who provided much of the inspiration for this project.

${ }^{1}$ P. K. Schelling and T. Le, J. Appl. Phys. 112, 083516 (2012).

${ }^{2}$ M. J. Gillan, "A simulation model for hydrogen in palladium: I. Singleparticle dynamics,” J. Phys. C 19, 6169 (1986).

${ }^{3}$ M. J. Gillan, "A simulation model for hydrogen in palladium: II. Mobility and thermotransport,” J. Phys. C 20, 521 (1987).

${ }^{4}$ A. B. Lidiard, Handb. der Physik (Springer Verlag, Berlin, 1957), Vol. 20, p. 246.

${ }^{5}$ J. H. Irving and J. G. Kirkwood, "The statistical mechanical theory of transport processes. IV. The equations of hydrodynamics," J. Chem. Phys. 18, 817 (1950).

${ }^{6}$ R. E. Howard and A. B. Lidiard, "Matter transport in solids," Rep. Prog. Phys. 27, 161 (1964).

${ }^{7}$ M. S. Green, "Markoff random processes and the statistical mechanics of time-dependent phenomena. 2. Irreversible processes in fluids," J. Chem. Phys. 22, 398 (1954).

${ }^{8}$ R. Kubo, "Statistical-mechanical theory of irreversible processes. 1. General theory and simple applications to magnetic and conduction problems,” J. Phys. Soc. Jpn. 12, 570 (1957).

${ }^{9}$ G. V. Paolini and G. Ciccotti, "Cross thermotransport in liquid mixtures by nonequilibrium molecular dynamics,” Phys. Rev. A 35, 5156 (1987).

${ }^{10}$ D. MacGowan and D. J. Evans, "Heat and matter transport in binary liquid mixtures," Phys. Rev. A 34, 2133 (1986).

${ }^{11}$ R. J. Bearman and J. G. Kirkwood, "Statistical mechanics of transport processes. XI. Equations of transport in multicomponent systems," J. Chem. Phys. 28, 136 (1958). 